\section{AB1301 IMMUNE REGULATION THERAPY PROMOTED THE RECANALIZATION OF CORONARY ARTERY IN A PATIENT WITH ANTIPHOSPHOLIPID SYNDROME: A CASE REPORT AND LITERATURE REVIEW}

Bin Wang, LI Fang, Chong Gao, LI Xiao-Feng. Second Hospital of Shanxi Medical University, Taiyuan, China

Background: Thrombosis is considered as a major feature of antiphospholipid syndrome (APS). Once formed, thrombosis will threaten life and long-term oral anticoagulant therapy is required. In addition, some patients underwent percutaneous coronary intervention $(\mathrm{PCI})$, but in-stent restenosis (ISR) is the most common complication of $\mathrm{PCl}$. There is currently insufficient treatment for such patients. Because APS is an autoimmune disease, we developed a new therapy (Sirolimus based immunomodulatory) to treat a APS patients with ISR after $\mathrm{PCI}$.

Objectives: Assess the degree of stenosis of the various branches of the coronary arteries, and to observe the changes in the number of regulatory $T$ cells (Tregs) in the peripheral blood of patients.

Methods: An APS patient with coronary artery involvement was found instent restenosis after implantation of the stent. After sirolimus and other immunomodulatory treatments, coronary angiography and coronary CTA were performed to assess the degree of stenosis of the various branches of the coronary arteries, and to observe the changes in the number of regulatory $\mathrm{T}$ cells (Tregs) in the peripheral blood of patients.

Results: After 6 months of immunomodulation therapy, the patient did not have clinical symptoms such as chest tightness and tightness. Four consecutive coronary angiography and coronary CTA (dual source) showed disappearance of left main plaque. Stenosis, the degree of stenosis in the anterior descending stent was reduced from $90 \%$ to $66 \%$; the degree of right coronary stenosis was reduced from $50 \%$ to $30 \%$, new stenosis and new thrombosis did not occur in other branches. The absolute number of Treg cells in peripheral blood gradually increased from 34.41 to 50.83 cells/ $\mu \mathrm{L}$.

Conclusion: Sirolimus is an inhibitor of mTOR that is a serine/threonine kinase in two different complexes (mTORC1 and mTORC2) to promote cell proliferation and neovascularization. Antiphospholipid antibodies activate the mTOR pathway in endothelial cells in culture. When pretreated with mTOR inhibitors prevents this process from occurring. Sirolimus specifically inhibits the mTOR pathway and prevents neointimal hyperplasia and neovascularization in the coronary arteries. In ASP patients with coronary artery involvement, the reduction of Treg cells may also be an important cause of immune tolerance deficiency. Sirolimus also increased the number of Treg cells. If early use of immunomodulators such as sirolimus for prophylaxis, it may prevent re-stenosis after stenting. The successful treatment of this patient also provided us with new diagnostic and therapeutic ideas.

\section{REFERENCES}

[1] Chinese Medical Association Rheumatology Branch. Guidelines for the diagnosis and treatment of antiphospholipid syndrome. Chinese Journal of Rheumatology, June 2011, Vol. 15, No. 6

[2] Nayak AK,Komatireddy G.Cardiac manifestations of the antiphospholipid antibody syndrome: a review. Mo Med.2002 Apr;99(4):171-8.

[3] Liu J, Liu Y, Jia K, et al. Clinical analysis of lectin-like oxidized low-density lipoprotein receptor-1 in patients with in-stent restenosis after percutaneous coronary intervention.Medicine (Baltimore).2018 Apr;97(17):e0366.

[4] Watanabe R, Wei L, Huang J. mTOR signaling, function, novel inhibitors, and therapeutic targets. J Nucl Med.2011 Apr;52(4):497-500.

[5] Van Laecke S, et al. Inhibition of the mTORC pathway in the antiphospholipid syndrome. N Engl J Med.2014 Jul 24;371(4):303-12.

Disclosure of Interests: None declared DOI: 10.1136/annrheumdis-2019-eular.5448

\section{AB1302 SOCIAL MEDIA USE IN SYSTEMIC LUPUS ERYTHEMATOSUS IMPACT ON PREVALENCE OF ANXIETY AND DEPRESSION}

Peiwen $\mathrm{Yi}^{1}$, Zhaohui Zheng ${ }^{1}$. The First Affiliated Hospital of Zhengzhou University, Zhengzhou, China

Background: The psychological problems of patients with Systemic lupus erythematosus (SLE) have been widely concerned. Studies found that the SLE patients showed a prevalence of depression in SLE cohorts ranging from $17 \%$ to $75 \%$, and anxiety being $6 \%-52 \%[1,2]$. Factors contributing to mood disorders are various[3]. As a nontraditional form of media, social media has become increasingly popular worldwide[4]. Previous study has demonstrated that social media use (SMU) plays a significant role in adolescents' psychological functioning[5-8], whether SMU could impact the mental health of SLE patients, no previous finding of SMU on SLE patients is reported.

Objectives: We aimed to explore the potential association of the patterns of social media use (SMU) with anxiety and depression in patients with systemic lupus erythematosus (SLE).

Methods: The relevant data were collected from 402 SLE patients aged 18-60 years in the First Affiliated Hospital of Zhengzhou University between July 2018 and October 2018 for subsequent analysis. Demographic and clinical characteristics, the patterns of SMU, the reasons and barriers to SMU were recorded. Anxiety and depression were assessed using hospital anxiety and depression scale (HADS). Adjusted estimates of association were derived using logistic regression.

Results: The incidence of anxiety and depression were $41.1 \%$ and $28.4 \%$ respectively among SLE patients with a mean score of $6.71 \pm 3.65$ and $5.45 \pm 3.86$ in each subscale. Patients without social media were more likely than those with social media to develop anxiety or depression ( $P$ $0.05, P 0.01)$. A correlation was found between the frequency of SMU and the incidences of anxiety $(P 0.05)$ and depression $(P 0.001)$, besides, the presences of depression also had a association with the duration for SMU ( $P$ 0.05)

Conclusion: Depression and anxiety are prevalent in patients with SLE and high frequency of SMU may decline the risks of anxiety and depression, to educate patients use social media properly may result in alleviation of anxious and depressive symptoms in patients with SLE.

\section{REFERENCES}

[1] Macedo EA, Appenzeller S, Costallat LTL. Depression in systemic lupus erythematosus: gender differences in the performance of the Beck Depres sion Inventory (BDI), Center for Epidemiologic Studies Depression Scale (CES-D), and Hospital Anxiety and Depression Scale (HADS). Lupus 2018;27(2):179-89.

[2] Jolly M, Sequeira W, Block JA, et al. Sex Differences in Quality of Life in Patients With Systemic Lupus Erythematosus. Arthritis Care Res (Hobo ken) 2018

[3] Tay SH, Cheung PP, Mak A. Active disease is independently associated with more severe anxiety rather than depressive symptoms in patients with systemic lupus erythematosus. Lupus 2015;24(13):1392-9.

[4] Nikiphorou E, Studenic P, Ammitzboll CG, et al. Social media use among young rheumatologists and basic scientists: results of an international survey by the Emerging EULAR Network (EMEUNET). Ann Rheum Dis 2017;76(4):712-15

[5] Escobar-Viera CG, Shensa A, Bowman ND, et al. Passive and Active Social Media Use and Depressive Symptoms Among United States Adults. Cyberpsychol Behav Soc Netw 2018;21(7):437-43.

[6] Shensa A, Escobar-Viera CG, Sidani JE, et al. Problematic social media use and depressive symptoms among U.S. young adults: A nationally-representative study. Soc Sci Med 2017:182:150-57.

[7] Berryman C, Ferguson CJ, Negy C. Social Media Use and Mental Health among Young Adults. Psychiatr Q 2018;89(2):307-14

[8] Griffiths S, Murray SB, Krug I, et al. The Contribution of Social Media to Body Dissatisfaction, Eating Disorder Symptoms, and Anabolic Steroid Use Among Sexual Minority Men. Cyberpsychol Behav Soc Netw 2018;21 (3):149-56

Disclosure of Interests: None declared

DOI: 10.1136/annrheumdis-2019-eular.1807

\section{AB1302B $\quad$ CHRONIC KIDNEY DISEASE IN RHEUMATOID ARTHRITIS: PREVALENCE AND RISK FACTORS}

Natalia Chebotareva, Tatyana Androsova, Ekaterina Kuznezova. Sechenov First Moscow Medical University, Moscow, Russian Federation

Background: The problem of chronic kidney diseases (CKD) in rheumatoid arthritis (RA) patients have not been fully studied. The role of biological therapy (BT) is poorly understood

Objectives: Aim of the study was to examine the prevalence, risk factors and histological variants of CKD in RA, and impact of pharmacotherapy Methods: 135 patients with RA from 2013 to 2018 were enrolled in this study. Age, gender, duration of RA, drug therapy, ESR, CRP, DAS28, renal function, proteinuria (PU), histological variants were analyzed. Arterial hypertension, weight index, serum lipids and glucose levels were also assessed.

Results: The incidence of CKD in RA was 30.4\% (41 from 135). CKD 3-4 stages with eGFR $<60 \mathrm{ml} / \mathrm{min} / 1.73 \mathrm{~m}^{2}$ was detected in $14.8 \%$ 